\title{
The Physics of Microfabrication
}




\section{The Physics of Microfabrication}

\section{Ivor Brodie and Julius J. Muray}

SRI International (formerly Stanford Research Institute) Menlo Park, California 


\section{Library of Congress Cataloging in Publication Data}

Brodie, Ivor.

The physics of microfabrication.

Bibliography: p.

Includes index.

1. Microelectronics. 2. Thin-film circuits. I. Muray, Julius J. II. Title. TK7874.B73 $621.381^{\prime 7}$

ISBN 978-1-4899-2162-8 ISBN 978-1-4899-2160-4 (eBook)

DOI 10.1007/978-1-4899-2160-4

First Printing - May 1982

Second Printing-November 1983

Third Printing - June 1984

Fourth Printing-April 1987

(C) Springer Science+Business Media New York 1982

Originally published by Plenum Press, New York in 1982

Softcover reprint of the hardcover 1st edition 1982

\section{All rights reserved}

No part of this book may be reproduced, stored in a retrieval system, or transmitted in any form or by any means, electronic, mechanical, photocopying, microfilming, recording, or otherwise, without written permission from the Publisher 


\section{Preface}

The Physical Electronics Department of SRI International (formerly Stanford Research Institute) has been pioneering the development of devices fabricated to submicron tolerances for well over 20 years. In 1961, a landmark paper on electron-beam lithography and its associated technologies was published by $\mathrm{K}$. R. Shoulders $\dagger$ (then at SRI), which set the stage for our subsequent efforts in this field. He had the foresight to believe that the building of such small devices was actually within the range of human capabilities.

As a result of this initial momentum, our experience in the technologies associated with microfabrication has become remarkably comprehensive, despite the relatively small size of our research activity. We have frequently been asked to deliver seminars or provide reviews on various aspects of microfabrication. These activities made us aware of the need for a comprehensive overview of the physics of microfabrication. We hope that this book will fill that need.

While there is a special emphasis on silicon microcircuit technology, this book is intended as an introduction for all engineers, scientists, and graduate students who are considering making something very small. The book may also serve as a source of review material for experienced workers in this extensive field, although no specialty is treated exhaustively. Though we have no desire to overlook anyone's contribution, we recognize that we may have failed to reference works or papers of significance. Any oversight is unintentional, and we would be pleased to receive suggestions for the improvement of any of the topics discussed. 
We wish to acknowledge the assistance provided by past and present members of the Physical Electronics Department scientific staff who have directly or indirectly helped us in the preparation of this book, especially Donald R. Cone, Eugene R. Westerberg, Charles A. Spindt, John B. Mooney, Robert A. Gutcheck, Arden Sher, T. E. Thompson, and John Kelly. We appreciate the support of SRI International through our Laboratory Director, Dr. Fred J. Kamphoefner, and our Division Executive Director, Earle D. Jones, in allowing us to use SRI facilities and materials.

We also wish to express our appreciation to our secretary, Joyce Garbutt, for carrying out the arduous task of typing the manuscript and to our publications group under the direction of Jack Byrne for editing, revisions, and illustrations. We would also like to thank Plenum Press for commissioning this book, and, finally, we would like to thank our wives and families for their patience during the tribulations encountered in the preparation of this book.

I. Brodie

J. J. Muray

Menlo Park, California

April 1981 


\section{Introduction}

Machines are devised for two basic purposes: to liberate humans from drudgery, and to help perform useful and interesting tasks that we would otherwise be unable to do. As far as size is concerned, machines may be classified into two general types-those with dimensions determined by their function and those whose size is limited only by economic factors. For instance, the dimensions of a plough are determined by the depth and width of the furrow it is to make, but the physical size of the motor that drives the plough or the size of the guidance mechanism that allows a desired ploughing pattern to be maintained are arbitrary and unrelated to the size of the furrow. However, the weight, power consumption, and cost of these ancillary devices are relevant to determining the economics of ploughing.

Both function and economics have supplied the driving forces toward making ever-smaller devices. In particular, the functional need to increase speed and the economic need to reduce the cost of binary storage elements for digital computers have led to the reduction in the size of such elements. What formerly required a flip-flop circuit using two tubes with a largest dimension of perhaps $5 \mathrm{~cm}$, and a cost measured in dollars, has now been replaced with an MOS memory element with a largest dimension of perhaps $15 \mu \mathrm{m}$, and a cost measured in millicents.

The continuing demand for inexpensive electronic circuits to feed the second industrial revolution has created the rapidly growing microelectronics industry. The economic pressure for lower cost and lower power consumption and the functional need for higher operational speeds have led to ever larger scales of integration and ever smaller sizes of circuit elements. New fabrication technologies have been and continue to be developed to satisfy the demands of this industry. These technologies include the following: deposition of thin films 
by sputtering and vacuum evaporation; pattern generation by optical, X-ray, and electron-beam lithographic techniques; and development of appropriate resist materials and methods of applying resists. Devices are routinely made with films as thin as $0.1 \mu \mathrm{m}$, with lateral dimensions as small as $5 \mu \mathrm{m}$ and with an edge sharpness on the order of $0.5 \mu \mathrm{m}$. With some of the technologies currently being developed, lateral dimensions of $0.5 \mu \mathrm{m}$ and edge sharpnesses of $0.1 \mu \mathrm{m}$ or less are being contemplated.

In addition to providing much of the technology required for fabricating microstructures, the incredible achievements of the microelectronic industry have also removed many of the psychological barriers to thinking small. It is awesome to consider that today's $\$ 500$ microcomputer has more memory than the first large electronic computer, ENIAC, yet it occupies $1 / 30,000$ of the volume, costs $1 / 10,000$ as much, performs its operations 20 times faster, and consumes the power of a light bulb rather than that of a locomotive. Moreover, we have not yet reached the practical limits to microminiaturization using currently available technologies.

The development of microcircuitry and its related technologies has encouraged people working in widely diverse disciplines to seriously consider making microstructures that would be useful in their fields. For example, in the biological area we may consider fabricating membranes and filters for artificial lungs and kidneys, artificial retinas and cochleas, and eventually even artificial cells. In the optical area, the ability to fabricate minute lenses, prisms, and other optical elements has given rise to integrated optical devices that are currently revolutionizing communications science. In the area of instrumentation we may visualize ultrasmall devices to probe, and manipulate with everincreasing sensitivity and precision.

Furthermore, we must remember that most of the microstructures we build today are "micro" only in the sense that their physical descriptions differ from those of bulk devices. They are still "macro" in the sense that they are large compared with atoms, and their dimensions are usually limited by some characteristic length associated with the physical principles by which they operate. Characteristic lengths, such as wavelength, Debye screening distances, or depletion depths, are intrinsically large compared with atomic distances, so that the technologies developed for fabricating these devices need not aspire to atomic resolution.

The technologies currently in use are largely based on removing material from a larger block, much as a sculptor carves a statue. In the more distant future we may look forward to molecular engineering technologies ${ }^{(1)} \dagger$ that will 
enable us to construct our devices atom by atom, much as a builder constructs a house brick by brick. It may be argued that molecular engineering has already been employed for simple applications, such as monomolecular film devices $^{(2,3)}$ and molecular beam epitaxy. ${ }^{(4)}$

In living cells, nature has given us extraordinarily diverse and intricate devices operating on the molecular level that we may attempt to emulate. The mystery of how these devices function must be solved before molecular engineering can become a reality. Gaining this understanding will require the use of new microinstrumentation techniques ${ }^{(5-8)}$ and a more sophisticated physical interpretation $^{(9)}$ of the measurements so obtained.

The driving forces toward microminiaturization will be dissipated only when the potential of molecular engineering has been fully exploited. However, long before that time arrives we may anticipate that the variety and complexity of microdevices will increase substantially, contributing significantly both to the well-being of mankind and our understanding of nature.

This book is directed to those well educated in an applied science or engineering but who are unfamiliar with all or some of the technologies used in microfabrication. Its purpose is to acquaint the reader with a basic physical understanding of the tools, technologies, and physical models needed to build, analyze, and understand the mechanism of microdevices, to see what physical limitations may be expected, to put numbers to the magnitudes involved, and to provide a guide to the extensive literature available. No attempt has been made to review all the microdevices that are being used or developed today; instead, we have emphasized a particular class of microelectronic devices based on planar silicon technology to illustrate how the techniques of microfabrication may be applied. We have chosen this class of devices because they have had such a major impact on our everyday life and economy and have provided impetus for the evolution of microfabrication technologies.

Our intention, however, is to be more general than required simply to meet the needs of those interested in microcircuit fabrication. We also wish to convince workers in other fields that building microstructures applicable to their needs may not be as difficult as they have imagined.

We have attempted to provide a bibliography that will be useful to the reader who wishes to follow up on a particular subject or who is interested in the historical aspects.

As a general rule, we have used mks units in the equations, unless some other units are specified. We have found that workers in a given specialty tend to develop their own mix of units that are used in common parlance. We have used their units, at least for educational purposes, as appropriate.

In Chapter 1 we present a preliminary survey of the subject matter. Our 
aim here is to introduce the reader to the types of devices that are microfabricated, particularly planar silicon devices, and provide a first look at the ways in which they are made. Those unfamiliar with microelectronics will also be able to pick up some of the jargon and the "alphabet soup" used to describe the subject matter.

We realize that Chapter 2 on particle beams may be heavy going for the reader. However, we believe that the effort to understand the physics involved in producing, focusing, and deflecting particle beams and their interactions with solid surfaces will be well worthwhile, since so much of the subsequently described technologies are based on these principles. Furthermore, this relieves the subsequent chapters from entering involved descriptions of details that could detract from the main topic under discussion.

Microdevice technology is heavily dependent on our ability to deposit and etch thin films of controlled physical and chemical properties. Chapter 3 reviews this important but arduous field.

While thin films have enabled one dimension of a device to be attained with astounding precision, the other two dimensions have proved to be more difficult. In Chapter 4 we discuss the technologies used to generate two-dimensional patterns in thin films, noting how they have improved to the point where patterns with micron-sized linewidths and a submicron edge sharpness can be routinely generated.

In Chapter 5 some important topics associated with silicon microcircuitry, namely, epitaxy, oxidation, doping, and annealing, are reviewed in more detail.

Having made a microdevice, we may need to "see" it and perform various measurements on it; such topics are discussed in Chapter 6.

Finally, in Chapter 7 we discuss the practical and theoretical limitations on microdevice fabrication and try to anticipate what the future might reasonably have in store in the way of microdevices. 


\section{Contents}

Introduction

vii

\section{Chapter 1 \\ PRELIMINARY SURVEY}

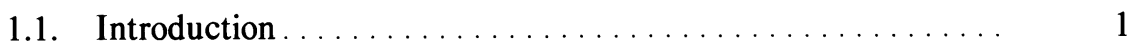

1.2. Microelectronic Devices ........................ 4

1.3. Planar Technologies Used in Fabricating Microelectronic Devices.................................. 13

1.3.1. Epitaxy ............................ 16

1.3.2. Oxidation . . . . . . . . . . . . . . . . . . . . 19

1.3.3. Lithographies ........................ 23

1.3.3.1. Photolithography .................. $\quad 24$

1.3.3.2. Projection Printing .................. $\quad 26$

1.3.3.3. Electron-Beam Lithography . . . . . . . . . . . $\quad 28$

1.3.3.4. X-Ray Lithography ................ $\quad 30$

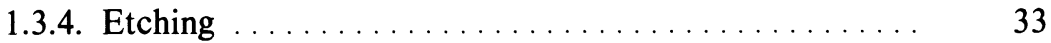

1.3.4.1. Chemical Etching . . . . . . . . . . . . . $\quad 33$

1.3.4.2. Anisotropic Etching .................. $\quad 34$

1.3.4.3. Ion Etching . . . . . . . . . . . . . . . . . . . $\quad 37$

1.3.4.4. Plasma Etching $\ldots \ldots \ldots \ldots \ldots \ldots \ldots . . \ldots \ldots$

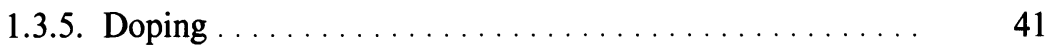

1.3.5.1. Diffusion ..................... $\quad 42$

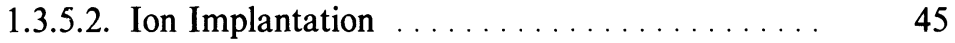

1.3.5.3. Neutron Transmutation Doping (NTD) ..... $\quad 50$ 
1.3.6. Thin-Film Deposition . . . . . . . . . . . . . . . . . $\quad 51$

1.3.6.1. Cathode Sputtering ................. 51

1.3.6.2. Vacuum Evaporation ................. 51

1.3.7. Interconnect Materials ..................... 53

1.3.8. Summary of Process Steps in Planar Technology ..... $\quad 55$

1.4. Microstructures for Other Applications . . . . . . . . . . . . 57

1.4.1. Physics ............................ 58

1.4.1.1. Integrated Optics ................. $\quad 58$

1.4.1.2. X-Ray Optics . . . . . . . . . . . . . . . . . $\quad 62$

1.4.1.3. Superconducting Junctions $\ldots \ldots \ldots \ldots \ldots . \ldots 2$

1.4.2. Electron and Ion Sources . . . . . . . . . . . . . . . . . 65

1.4.3. Materials Science . . . . . . . . . . . . . . . . . . . $\quad 69$

1.4.4. Unconventional Electronic Devices . . . . . . . . . . . . 71

1.4.4.1. Particle-Beam Memories ............... 71

1.4.4.2. Nonvolatile Semiconductor Memory Devices . . $\quad 74$

1.4.4.3. Charge-Trapping Devices .............. $\quad 74$

1.4.4.4. Floating-Gate Devices . . . . . . . . . . . $\quad 76$

1.4.4.5. Topologically Structured Thin Films . . . . . . $\quad 76$

1.4.5. Biology . . . . . . . . . . . . . . . . . . . . $\quad 78$

Chapter 2

PARTICLE BEAMS: SOURCES, OPTICS, AND INTERACTIONS

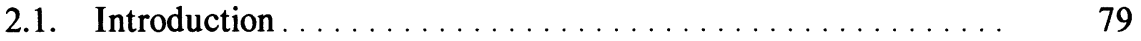

2.2. Electron Sources ....................... 81

2.2.1. Elementary Theory of Thermionic and Field Emission. . 81

2.2.2. Cathode Requirements .................... $\quad 87$

2.2.3. Practical Cathodes ..................... $\quad 90$

2.2.3.1. Thermionic Cathodes ................. $\quad 90$

2.2.3.2. Field-Emission Cathodes ............... $\quad 92$

2.3. Characteristics and Design of Electron Guns ............ 94

2.3.1: Gun Requirements and Limitations .............. 94

2.3.2. Image Formation . . . . . . . . . . . . . . . $\quad 97$

2.3.3. Beam Characteristics ..................... 100

2.3.4. Space-Charge Effects . . . . . . . . . . . . . . . . 105

2.3.5. Aberrations ........................ 107

2.3.5.1. Spherical Aberration ................. 107

2.3.5.2. Chromatic Aberration ................ 109

2.3.5.3. Astigmatism .................. 110 
2.3.5.4. Diffraction ................... 110

2.3.5.5. Defocusing Due to Beam Energy Spread ..... 111

2.3.6. Gun Structures . . . . . . . . . . . . . . . . . . . 113

2.3.7. Gun Optics .......................... 118

2.4. Ion Sources ........................... 123

2.4.1. Background ........................ 123

2.4.2. Electron-Impact Ionization Sources . . . . . . . . . . . 125

2.4.3. Plasmatrons .......................... $\quad 130$

2.4.4. Field-Ionization Sources. . . . . . . . . . . . . . . . . . 131

2.4.5. Liquid Field-Ionization Sources ............... 135

2.4.6. Space-Charge Effects . . . . . . . . . . . . . . . . . . . 137

2.5. Components for Electron and Ion Optics ............. 144

2.5.1. Electrostatic Lenses and Mirrors . . . . . . . . . . . . . . 144

2.5.2. Magnetic Lenses . . . . . . . . . . . . . . . . . . . . . . 157

2.5.3. Energy-Analyzing and Selecting Devices ......... 162

2.5.4. Image Aberrations . . . . . . . . . . . . . . . . . 166

2.5.4.1. Geometric Aberrations $\ldots \ldots \ldots \ldots \ldots \ldots .6166$

2.5.4.2. Electronic Aberrations . . . . . . . . . . . . $\quad 170$

2.5.5. Multiple-focusing Elements . . . . . . . . . . . . . 172

2.5.6. Deflection Systems . . . . . . . . . . . . . . . . . 179

2.6. Electron Interactions $\ldots \ldots \ldots \ldots \ldots \ldots \ldots \ldots \ldots \ldots \ldots$

2.6.1. Electron-Solid Interactions . . . . . . . . . . . . . 186

2.6.1.1. Introduction . . . . . . . . . . . . . . 186

2.6.1.2. Electron Transmission Through Thin Films ... 188

2.6.1.3. Elastic Scattering . . . . . . . . . . . . . . $\quad 188$

2.6.1.4. Beam Spreading in Thin Films . . . . . . . . . . 190

2.6.1.5. Inelastic Scattering . . . . . . . . . . . . . . 191

2.6.2. Energy-Loss Mechanisms . . . . . . . . . . . . . . . 193

2.6.2.1. Electron Range ...................... 195

2.6.2.2. Backscattered Electrons . . . . . . . . . . . . . $\quad 199$

2.6.2.3. Secondary Electrons.................. 201

2.6.2.4. X-Ray Emission ..................... . 204

2.6.3. Physical Effects in the Target ................ 206

2.6.3.1. Polymerization: Bond Breaking . . . . ..... 206

2.6.3.2. Thermal Effects . . . . . . . . . . . . . . . $\quad 208$

2.6.3.3. Current Injection ................. 209

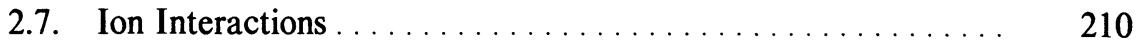

2.7.1. Introduction ....................... 210

2.7.2. Energy-Loss Mechanisms ................... 213

2.7.2.1. Nuclear Stopping . . . . . . . . . . . . . . $\quad 213$

2.7.2.2. Electronic Stopping . . . . . . . . . . . . . . . . . 214 
2.7.2.3. Range ........................ 215

2.7.2.4. Channeling .................. 216

2.7.2.5. Secondary Processes................. $\quad 217$

2.7.2.5.1. Sputtering .............. 217

2.7.2.5.2. Inelastic Processes ........... 218

2.7.2.5.3. Electron Emission . . . . . . . . . . . 218

2.7.2.5.4. X-Ray Emission .............. 219

2.8. Photon Sources and Interactions .................. 219

2.8.1. X-Ray Sources for Lithography ............... 219

2.8.1.1. Electron Bombardment Sources .......... $\quad 220$

2.8.1.2. Plasma Sources ................... $\quad 220$

2.8.1.3. Synchrotron Radiation . . . . . . . . . . . . . $\quad 220$

2.8.2. Light Sources ...................... 220

2.8.2.1. Laser Sources for Annealing . . . . . . . . . . $\quad 220$

2.8.2.2. Arc Lamps for Photolithography .......... 221

2.8.3. Photon Interactions . . . . . . . . . . . . . . . . . . 222

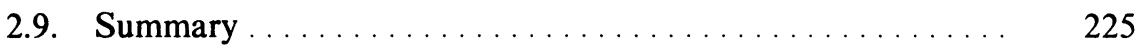

\section{Chapter 3 \\ THIN FILMS}

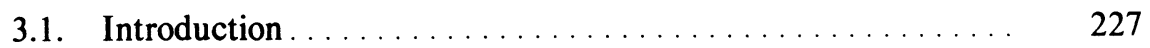

3.2. Deposition Technologies . . . . . . . . . . . . . . . . . . 227

3.2.1. Overview ........................... 227

3.2.2. Evaporation in Ultrahigh Vacuum . . . . . . . . . 229

3.2.3. Sputtering ....................... 235

3.2.3.1. Sputter Rate, Angular, and Energy

Distributions ...................... 236

3.2.3.2. Implementation of Sputtering for Thin-Film

Deposition ........................ 238

3.2.4. Chemical Vapor Deposition ................... 243

3.2.5. Organic Thin Films ...................... 245

3.2.6. Surface Preparation . . . . . . . . . . . . . . . . . $\quad 248$

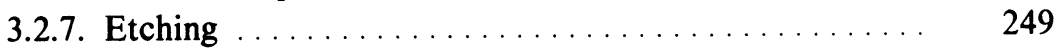

3.2.7.1. Chemical Etching . . . . . . . . . . . . . . 249

3.2.7.2. Sputter Etching . . ................ $\quad 252$

3.2.7.3. Reactive Plasma Etching ................ 253

3.2.8. Thickness and Rate Measurement ............. 255 
3.3. Nucleation, Composition, Morphology, and Structure ..... 257

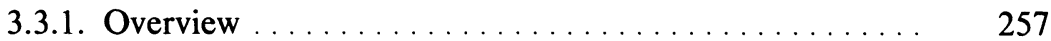

3.3.2. Nucleation and Initial Growth ............... 259

3.3.3. Postnucleation Growth Processes . . . . . . . . . . . . . 262

3.3.4. Structure and Composition ................... 263

3.3.4.1. Morphology ................... 263

3.3.4.2. Crystallinity .................... 263

3.3.4.3. Lattice Constants . . . . . . . . . . . . . . . . . 263

3.3.4.4. Composition ...................... 264

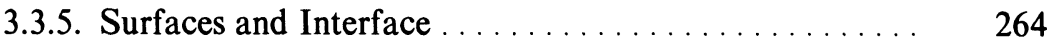

\section{Chapter 4 \\ PATTERN GENERATION}

4.1. Introduction . . . . . . . . . . . . . . . . . . . . . . 267

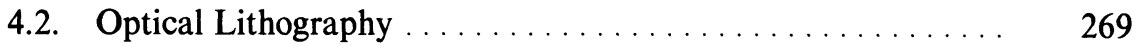

4.2.1. Contact Printing ........................ $\quad 269$

4.2.2. Proximity Printing $\ldots \ldots \ldots \ldots \ldots \ldots \ldots \ldots . \ldots . \ldots . \ldots 270$

4.2.3. Projection Printing ..................... 271

4.3. The Physics of Photolithography . . . . . . . . . . . . . . 278

4.3.1. Positive Photoresist Exposure . . . . . . . . . . . . . . 278

4.3.2. Resist Characterization .................... 281

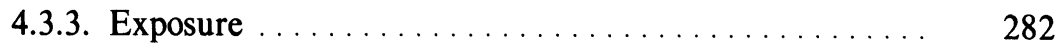

4.3.4. Interference Effects . . . . . . . . . . . . . . . . . . . 284

4.3.5. Resist Development ...................... 288

4.3.6. Negative Photoresists . . . . . . . . . . . . . . . . . . . . 288

4.4. Projection Systems . . . . . . . . . . . . . . . . . . . 291

4.5. Holographic Lithography . . . . . . . . . . . . . . . . . . . 293

4.6. X-Ray Lithography ........................ $\quad 294$

4.6.1. The X-Ray Lithography System . . . . . . . . . . . . 294

4.6.2. X-Ray Sources . . . . . . . . . . . . . . . . . . . . . . . 297

4.6.3. Plasma Sources . . . . . . . . . . . . . . . . . . . . . . . . . 299

4.6.4. X-Ray Masks . . . . . . . . . . . . . . . . . . . . . . 300

4.6.5. X-Ray Resists . . . . . . . . . . . . . . . . . . . . . 304

4.6.6. Alignment . . . . . . . . . . . . . . . . . . . 305

4.6.7. Linewidth Control ........................ 306

4.7. Synchrotron Radiation for X-Ray Lithography . . . . . . . . . . 308

4.7.1. Introduction . . . . . . . . . . . . . . . . . . . . 308

4.7.2. Properties of Synchrotron Radiation . . . . . . . . . . . . . 308 


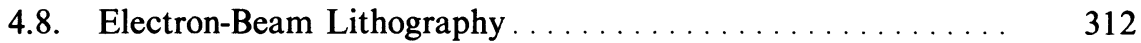

4.8.1. Introduction . . . . . . . . . . . . . . . . . 312

4.8.2. Major System Components .................. . 314

4.8.2.1. Projection Systems . . . . . . . . . . . . . . . . . $\quad 314$

4.8.2.2. Beam-Scanning Systems ............. 316

4.8.2.2.1. The Electron-Optical Column ..... 316

4.8.2.2.2. Beam Size and Shape.......... $\quad 319$

4.8.2.2.3. Pattern Generation ............ $\quad 320$

4.8.2.2.4. Stage Assembly ............. $\quad 322$

4.8.2.2.5. Registration............... $\quad 324$

4.8.3. Parallel-Scanning Systems . . . . . . . . . . . . . $\quad 326$

4.8.4. Electron-Beam Resists . . . . . . . . . . . . . . . . . $\quad 329$

4.8.4.1. Resist Characterization . . . . . . . . . . . . . . 329

4.8.4.2. Negative Resists .................. $\quad 332$

4.8.5. Cross-Sectional Profiles of Single-Scan Lines ....... 335

4.8.6. Development . . . . . . . . . . . . . . . . . . . 337

4.8.7. Proximity Effects . . . . . . . . . . . . . . . 338

4.8.8. Specifications for Electron-Beam Exposure Systems .... 338

4.9. Ion-Beam Lithography . . . . . . . . . . . . . . . . . . . . $\quad 339$

4.9.1. Introduction ....................... 339

4.9.2. Ion-Beam Resists . . . . . . . . . . . . . . . . . . . . . 344

4.9.3. Liquid-Metal (LM) Ion Sources . . . . . . . . . . . 346

4.9.4. Scanning Systems . . . . . . . . . . . . . . . 346

Chapter 5

SPECIAL PROCESSES DEVELOPED FOR MICROCIRCUIT TECHNOLOGY

5.1. Epitaxy and Oxidation . . . . . . . . . . . . . . . . $\quad 349$

5.1.1. Silicon Epitaxy . . . . . . . . . . . . . . . . . . . $\quad 349$

5.1.1.1. Growth Processes . . . . . . . . . . . . . . . . . 349

5.1.1.2. Indirect Processes . . . . . . . . . . . . . . . $\quad 350$

5.1.2. Liquid-Phase Epitaxy . . . . . . . . . . . . . . . . . 354

5.1.3. Molecular Beam Epitaxy (MBE) .............. $\quad 356$

5.1.4. Oxidation . . . . . . . . . . . . . . . . . . . . 358

5.1.4.1. The Basic Deal-Grove Model . . . . . . . . . . . 359

5.1.4.2. Crystal Orientation Effects ............. $\quad 360$

5.1.4.3. Impurity Doping Effects ............. 360 
5.1.4.4. High-Pressure Oxidation ............... $\quad 361$

5.1.4.5. Oxidation in $\mathrm{HCl}-\mathrm{O}_{2}$ Mixtures .......... 362

5.1.4.6. Oxide Charges ................... 363

5.2. Doping .............................. 367

5.2.1. Diffusion .......................... 367

5.2.1.1. Field-Enhanced Diffusion ............. $\quad 370$

5.2.1.2. Ionization-Enhanced Diffusion ........... 371

5.2.1.3. Energy Release-Enhanced Diffusion ........ 371

5.2.2. Ion Implantion . . . . . . . . . . . . . . . . . 372

5.2.2.1. Qualitative Features of Ion Implantation..... 373

5.2.2.2. Range Theory-Lindhard, Scharff, Schiott (LSS) ........................ $\quad 374$

5.2.2.3. Useful Approximations . . . . . . . . . . . . . . $\quad 375$

5.2.2.4. Projected Range ..................... $\quad 377$

5.2.2.5. Masking . . . . . . . . . . . . . . . . . . . . $\quad 379$

5.2.2.6. Channeling ................... 383

5.2.2.7. Implantation . . . . . . . . . . . . . . 383

5.2.2.8. Damage ..................... $\quad 385$

5.2.2.8.1. Heavy Ions-Sb, As . . . . . . . . 386

5.2.2.8.2. Light Ions-B . . . . . . . . . . 387

5.2.2.8.3. The Creation of Amorphous Material .................. 387

5.2.2.8.4. Damage Profiles . . . . . . . . . . . . $\quad 387$

5.2.2.8.5. The Location of Implanted Ions .... $\quad 390$

5.2.3. Radiation-Enhanced Diffusion . . . . . . . . . . . . . . 390

5.2.4. Recoil Phenomena ........................ 391

5.2.5. High-Dose Implantation Limits . . . . . . . . . . . . . . . 394

5.2.6. Device Applications . . . . . . . . . . . . . . . . . . . . 394

5.2.6.1. Bipolar Devices. . . . . . . . . . . . . . . . . . . $\quad 394$

5.2.6.2. Mos Devices . . . . . . . . . . . . . . . . . . 397

5.2.6.3. Resistors . . . . . . . . . . . . . . . . . . . . 398

5.2.7. Neutron Transmutation Doping . . . . . . . . . . . . 398

5.3. Laser-Assisted Processes . . . . . . . . . . . . . . . . . . . . . 401

5.3.1. Laser Annealing ....................... 401

5.3.2. Thermal Analysis of Laser Annealing . . . . . . . . . . 404

5.3.2.1. Adiabatic Limit . . . . . . . . . . . . . . . . . . 405

5.3.2.2. Diffusion Limit . . . . . . . . . . . . . . . . 406

5.3.3. Crystalline Conversion . . . . . . . . . . . . . . . . . . . 408

5.3.4. Laser-Assisted Diffusion . . . . . . . . . . . . . . . 408

5.3.5. Graphoepitaxy ........................... 409 
5.4. Electron-Beam-Assisted Processes . . . . . . . . . . . . . . . . . . 409

5.4.1. Electron-Beam Annealing ................... 409

5.4.2. Thermal Analysis of Electron-Beam Annealing ....... 410

5.5. Computer Simulation of Fabrication Processes .......... 412

\section{Chapter 6 \\ SUBMICRON MICROSCOPY AND MICROPROBES}

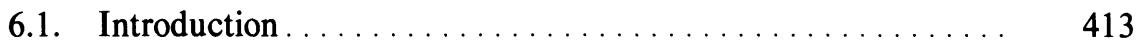

6.2. Transmission Electron Microscopes ................. 416

6.3. Scanning Electron Microscopes . . . . . . . . . . . . . 421

6.4. Electron-Reflection Microscopes . . . . . . . . . . . . . . . . 425

6.5. Electron Emission Microscopes . . . . . . . . . . . . . . . . 429

6.6. Field-Ion Microscopes . . . . . . . . . . . . . . . . . . . . 433

6.7. X-Ray and Ion Microprobes . . . . . . . . . . . . . 435

6.8. Laser Scanning ............................ 438

6.9. Scanning Acoustic Microscopes . . . . . . . . . . . . . . . 440

6.10 Device Characterization by Microscopy ............. 441

\section{Chapter 7 \\ FUTURE DIRECTIONS}

7.1. Limits and Trends-General . . . . . . . . . . . . . . . . . . 443

7.2. Limits for Mos Devices . . . . . . . . . . . . . . . . . . . . $\quad 445$

7.2.1. Scaling Laws .......................... $\quad 445$

7.2.2. Fundamental Limits . . . . . . . . . . . . . . . . . . . $\quad 449$

7.2.2.1. Quantum Limits . . . . . . . . . . . . . . . . . . . 449

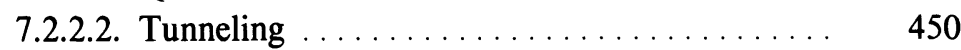

7.2.3. Material Limitations $\ldots \ldots \ldots \ldots \ldots \ldots \ldots \ldots . \quad 450$

7.2.4. Device Limits . . . . . . . . . . . . . . . . . . . . . . . . 451

7.3. Limits in Pattern Generation ................... $\quad 453$

7.3.1. Limits on Linewidths and Edge Sharpness . . . . . . . . 453

7.3.2. Resist Materials ........................ 454

7.3.3. Exposure Statistics ...................... 456

7.3.3.1. Electron-Beam Exposure . . . . . . . . . . . . . 456

7.3.3.2. X-Ray Resist Exposure ................ $\quad 459$

7.4. Summary ........................... 462 


\section{APPENDICES}

A. The Error Function and Some of Its Properties ........... 465

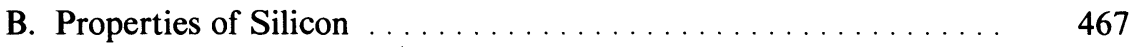

C. Useful Physical Constants in Microscience .............. 469

References ............................... $\quad 471$

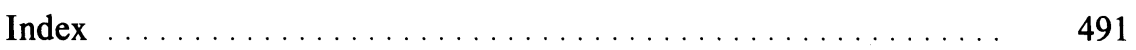

